\title{
Comparative Analysis of Simulation Time in Nonlinear and Harmonically Excited Pendulum and Duffing Oscillators
}

\author{
Salau T. A.O. ${ }^{1}$ and Ajide O.O. ${ }^{2}$ \\ Department of Mechanical Engineering, \\ University of Ibadan, Nigeria. \\ tao.salau@mail.ui.edu.ng \\ ooe.ajide@mail.ui.edu.ng ${ }^{2}$
}

\begin{abstract}
The motivation for the present study is derived from the fact that time mangaement is an integral part of good engineering practice. The present study investigated the quantification of the required computation time using two nonlinear and harmonically excited oscillators (Pendulum and Duffing) as case studies. Simulations with personal computer were effected for Runge-Kutta schemes (RK2, RK3, RK4, RK5, RK5M) and one blend (RKB) over thirty five thousand and ten excitation periods consisting the unsteady and steady solutions. The need for validation of the developed FORTRAN90 codes by comparing Poincare results with their conterpart from the literature informed the choice of simulation parameters. However, the simulation time was monitored at three lengths of excitation period (15000, 25000 and 35000) using the current time subroutine call command.

The validation Poincaré results obtained for all the schemes including RKB compare well with the counterpart available in the literature for both Pendulum and Duffing. The actual computation time increases with increasing order of scheme, but suffered a decrease for the blended scheme. The diffencerence in computation time required between RK5 and RK5M is negligible for all studied cases. The actual computational time for Duffing (5-33seconds) remain consistently higher for corresponding Pendulum (3-23seconds) with difference (2-10seconds). Interestingly, the quantitative difference between the corresponding normalised computation time for systems and schemes is insignificant. It is insensitive to systems and schemes and formed a simple average ratio $\{(1.0):(1.5):(2.0):(3.1):(3.1):(2.4)\}$ for RK2, RK3, RK4, RK5, RK5M and $\mathrm{RKB}$ respectively. It is concluded that the end justified the means provided that computation accuracy is assured using the higher order scheme (with higher computational time ratio).
\end{abstract}

Keywords: Simulation time, Nonlinear system, Harmonically excited system, Runge-Kutta schemes and Excitation period

\section{Council for Innovative Research}

Peer Review Research Publishing System

Journal: INTERNATIONAL JOURNAL OF COMPUTERS \& TECHNOLOGY

Vol 10, No 2

editor@cirworld.com

www.cirworld.com, member.cirworld.com 


\section{INTRODUCTION}

Computer simulations has been described as a process of designing a model of a real system , implementing the model as a computer program, and performing experiments with the model for the rationale of understanding the behaviour of the system, or evaluating strategies for the operation of the system (Classweb, 2013). There is no iota of doubt that time saving and management without compromise of its accuracy is crucial in the choice or acceptability of a numerical simulation technique. Finger and Uhlmann (1994) have demonstrated how the choice of a particular numerical method can help save a great deal of computational time. In their paper, the authors applied Runge-Kutta triples in estimating Poincaré for autonomous systems. The outcome of the study revealed that a remarkable computational time can be saved with Runge-Kutta triples by using an interpolation polynomial for dense output. Their research paper is indeed a great contribution to computer time management in simulation of dynamical systems. Haecheon and Parviz (1994) studied the effects of the computational time step on numerical solutions of turbulent flow. In turbulent channel flow computations; the largest computational time in wall units which led to the accurate prediction of turbulence statistics was estimated. It was concluded from the authors' paper that turbulence fluctuations can only be sustained by a cautious choice of computational time step. Ludovic et al (2002) proposed an automatic time stepping algorithm useful for numerical simulations of nonlinear dynamics. The authors learnt from research experience that constant step size strategies generally lead to divergence or extremely costly computations. This motivated the authors to initiate an algorithm that automatically takes decision in order to update the tangent matrix or stopping the iterations. The authors demonstrated that this technique has reduced the computational time cost using several real life industrial problems. This is no doubt a great contribution to researchers' efforts in saving the computer time required for numerical simulations. As part of efforts made in improving the computational time efficiency in the numerical solutions of the differential algebraic equations often encountered in the power system simulation, Siddhartha and James (2008) applied unsymmetrical multifrontal method to solve the differential equations. Simulation results showed that the method achieves enormous computational advantage when compared to the conventional Gaussian methods as well as other linear sparse solvers. The application of nonlinear dynamic models in economics and finance has experienced astronomical expansion in the last two decades (Diks et al, 2008). The authors developed a user friendly software package for nonlinear dynamics. The software is embedded with many nonlinear model features that are easy to use without the need for a compiler or additional software. The authors asserted that a shorter and more efficient representation within the software is capable of reducing the computational time. A study which utilized blend of phase plots ,time steps and adaptive time steps as well as the fifth order Runge-Kutta algorithms has been used by Salau and Ajide (2012) to study a harmonically excited Duffing oscillator dynamics. Findings of the study showed that there is favourable computational time with the use of fifth order Runge-Kutta method. The authors however concluded that a fourth order algorithm is the more appropriate method when very high accuracy is desired although with higher computational time. In an article written by Sett (2013), the need to reduce computer simulation time was emphasized. The authors satisfactorily describe a technique of running multiple simulations in parallel. The authors explained that it is possible to reduce the computational time it take to run multiple independent simulations by distributing simulation tasks among multiple processing cores with simulink and parallel computing too box. The overall simulation time can be further reduced by using MATLAB distributed computing server to run the simulations on a computer cluster.

The immense importance of Runge-Kutta method as numerical techniques in the study of nonlinear system characteristics cannot be overemphasized. Several investigations of the nonlinear dynamical behaviours under different scenarios have been carried out by researchers using Runge-Kutta techniques. A study which bothers on the implementation of fourth and fifth order Runge-Kutta was implemented for Rössler system (Nikolaos , 2009). The author utilized explicit MATLAB algorithm for the implementation of fourth and fifth orders Runge-Kutta. Using Rössler system of equations, results obtained showed that fifth order obviously gives smaller errors for a given running time when compared with the fourth order. In 2011, Murugesh et al applied one of the newly developed Runge-Kutta methods known as RK-Butcher algorithm in the simulation study of nonlinear singular system. The method was found to be efficient and very accurate when compared to the classical fourth order Runge-Kutta. The paper concluded that this newly developed RK-Butcher algorithm can easily be implemented in a digital computer and the simulation results can be obtained for any length of time with high precisions. In the very recent time, so many researchers utilized Runge-Kutta methods in the characterization of nonlinear dynamics systems [See: Jian and Huazhong (2013), Wang (2013), Mustafa et al 2013, Salau and Ajide (2013)].

Pendulum and Duffing oscillators that are harmonically excited are two of the numerous nonlinear mechanical systems that Runge-Kutta has been intensely used as numerical technique. Salau and Ajide (2013) utilized Runge-Kutta schemes in the study of fractal characteristics of harmonically excited pendulum using poincaré sectioning approach. The outcome of their study has shown the utility of the novel graphic plots as a dynamic systems characterizing tool for harmonically excited pendulum. Harmonically excited Pendulum and Duffing oscillators are specifically attracting some researchers' interests. This is because of their versatile engineering applications. Therefore, other methods are equally being explored as means of their characterization in addition to the popular Runge-Kutta methods. Liang and Feeny (2008) have studied the parametric identification of a chaotic based-excited double pendulum dynamics. The parametric identification of the chaotic system was examined through a careful recorded response experimental data. The unstable periodic orbits were extracted from the data and used in harmonic balance identification for the process. The authors' experimental results informed that the formulated identification algorithm for the system is highly successful. Chen et al (2012) examined the stochastistic stability of the harmonically and randomly excited Duffing oscillator. The obtained results from the Lyapunov exponent have shown the richness in the characteristics of harmonically excited Duffing oscillator. The effects of different damping mechanisms on the response of harmonically excited Duffing oscillators have been examined (Ivana et al, 2011). Results obtained have no doubt provided a significant platform for interested researchers in this field. 
From the foregoing, there is no doubt that avalanche of literature exists in the nonlinear characterization of the very important mechanical engineering systems such as harmonically excited Duffing oscillator and Pendulum by means of Runge-Kutta schemes as well other equally acceptable techniques. In spite of the landmark achievements in the characterization of these two nonlinear systems, an obvious lacuna exists. There is dearth of literature that bothers on the required computational time for different Runge-Kutta schemes using two nonlinear and harmonically excited oscillators (Pendulum and Duffing) as case studies. The importance of time saving and management in engineering practice cannot be over emphasized. The strong zeal of the authors in saving/managing the computer time required for numerical simulations of nonlinear dynamical systems immensely motivated the present paper.

\section{METHODOLOGY}

The second order governing equations for the oscillators (Pendulum and Duffing) were adapted from Gregory and Jerry (1990) and Dowell (1988) respectively. In equation (1) $q$ is the damping quality parameter, $g$ is the forcing amplitude, and $\omega_{D}$ is the drive frequency. Similarly, in equation (2) the damp coefficient is $\gamma$, the amplitude strength of harmonic excitation and excitation frequency are respectively $P_{o}, \omega$. The time $(\mathrm{t})$ is a common variable to the two equations.

$$
\begin{aligned}
& \frac{d^{2} \theta}{d t}+\frac{1}{q} \frac{d \theta}{d t}+\sin (\theta)=g \cos \left(\omega_{D} t\right) \\
& \ddot{x}+\gamma \dot{x}-\frac{x}{2}\left(1-x^{2}\right)=P_{o} \operatorname{Sin}(\omega t)
\end{aligned}
$$

The relevant transformation of equations (1) and (2) that will enable their Runge-Kutta simulation under the assumptions ( $\theta_{1}=$ angular displacement,$\theta_{2}=$ angular velocity,$\quad x_{1}=$ linear displacement and $x_{2}=$ linear velocity) yield respectively pair of first order differential equations ( $3 \& 4$ and $5 \& 6$ ).

$$
\begin{aligned}
& \dot{\theta}_{1}=\theta_{2} \\
& \dot{\theta}_{2}=g \cos \left(\omega_{D} t\right)-\frac{1}{q} \theta_{2}-\sin \left(\theta_{1}\right) \\
& \dot{x_{1}}=x_{2} \\
& \dot{x}_{2}=P_{o} \operatorname{Sin}(\omega t)-\gamma x_{2}+\frac{x_{1}}{2}\left(1-x_{1}^{2}\right)
\end{aligned}
$$

The present study utilised the under-listed popular constant operation time step Runge-Kutta schemes (codes in FORTRAN90) to simulate equations (3) to (6) from initial conditions $(0,0)$ through unsteady and steady solutions. The unsteady and steady simulations spanned ten thirty five thousand excitation periods respectively for each scheme investigated.

$>$ Second order Runge-Kutta (RK2)

$>$ Third order Runge-Kutta (RK3)

$>$ Fourth order Runge-Kutta (RK4)

$>$ Fifth order Runge-Kutta (RK5)

$>$ Butchers' (1964) Modified Fifth order Runge-Kutta (RK5M)

Uniform blend (driven with random seed value of 9876) Runge-Kutta (RKB=RK2+RK3+RK4+RK5+RK5M)

Steven and Raymond (2006) refer. The simulation predictor of the different Runge-Kutta schemes as function of derivatives $\left(K_{i}\right)$ evaluated at i-nodes (scheme specific) within the time step interval $(\Delta t=h)$ are given in equations (7) to (10). It is important to note that $y \leftarrow \theta_{1}, \theta_{2}$ for the Pendulum and $y \leftarrow x_{1}, x_{2}$ for the Duffing.

\subsection{Second order Runge-Kutta (RK2):}

$$
y_{i+1}=y_{i}+\frac{h}{2}\left\{K_{1}+K_{2}\right\}
$$

\subsection{Third order Runge-Kutta (RK3):}


$y_{i+1}=y_{i}+\frac{h}{6}\left\{K_{1}+4 K_{2}+K_{3}\right\}$

\subsection{Fourth order Runge-Kutta (RK4):}

$y_{i+1}=y_{i}+\frac{h}{6}\left\{K_{1}+2\left(K_{2}+K_{3}\right)+K_{4}\right\}$

\subsection{Fifth order Runge-Kutta Method (RK5):}

$y_{i+1}=y_{i}+\frac{h}{90}\left\{7 K_{1}+32 K_{3}+12 K_{4}+32 K_{5}+7 K_{6}\right\}$

The fifth order Runge-Kutta scheme and the Butchers' (1964) modified fifth order Runge-Kutta scheme shared the same predictor function as in equation (10). However the nodes specifications are different.

\subsection{Simulation Parameters}

The basic relevant properties of the laptop used for this study are as follow:

Processor: $\quad$ Intel (R) Pentium (R) Dual CPU T3400 @2.16GHz 2.17GHz

Memory (RAM): $2.00 \mathrm{~GB}$

\section{System type: 32-bit operating system}

The Pendulum and Duffing were simulated with $\left(q, g \omega_{D} \equiv 4,1.5,2 / 3\right)$ and $\left(\gamma, P_{o} \omega \equiv 0.168,0.21,1.0\right.$ ) respectively. These setting serve well for validation of the FORTRAN90 codes developed for the present study. The constant simulation time step is $h=T_{p} / 500$, where $T_{p}$ represent the excitation period.

The timer is the system clock which responds to a subroutine call command-CALL SYSTEM_CLOCK (ITIME).

\section{RESULTS AND DISCUSSIONS}

The Poincare section for the validation cases are given in figures 1 (a) and 1(b). The sections compare correspondingly well with the results of Gregory and Jerry (1990) and Dowell (1988). Furthermore all the schemes including the blended (RKB) return the same qualitative corresponding Poincare section, see sample in figures 2(a) and 2(b).

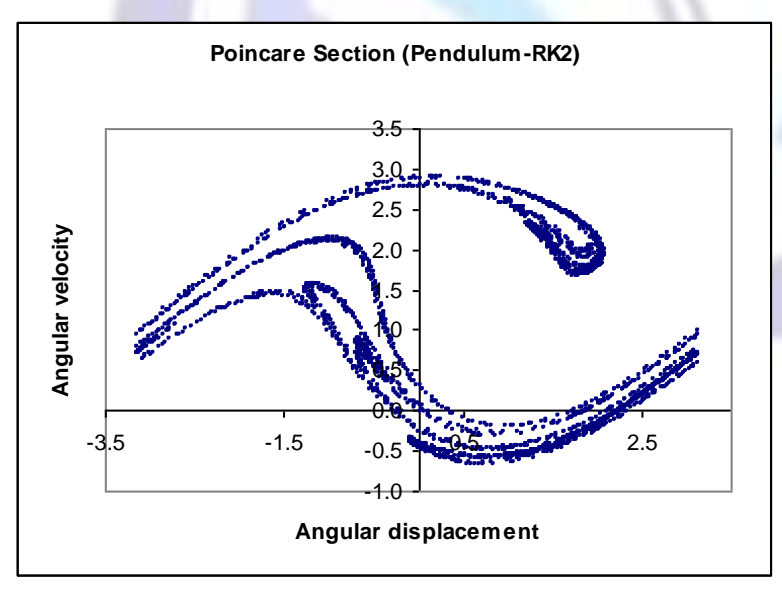

(a)

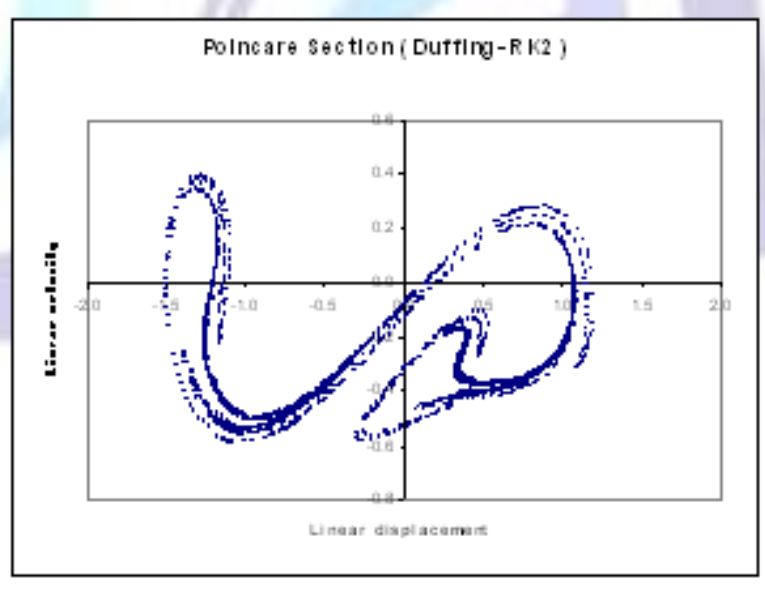

(b)

Figure 1: $\quad$ Poincare sections of Pendulum $\left(q, g \omega_{D} \equiv 4,1.5,2 / 3\right)$ and Duffing $\left(\gamma, P_{o} \omega \equiv 0.168,0.21,1.0\right.$ ) oscillators using second order Runge-Kutta scheme 


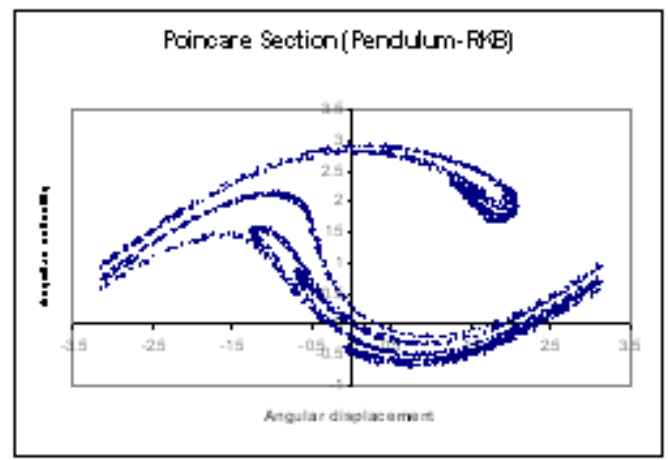

(a)

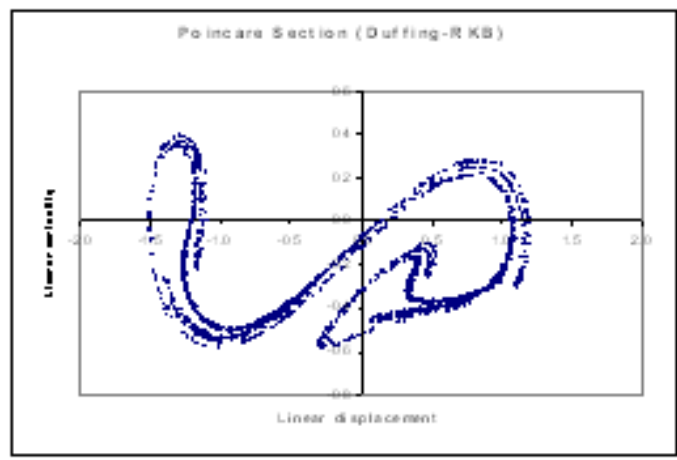

(b)

Figure 2:Poincare sections of Pendulum $\left(q, g \omega_{D} \equiv 4,1.5,2 / 3\right)$ and Duffing $\left(\gamma, P_{o} \omega \equiv 0.168,0.21,1.0\right)$ oscillators using the blended scheme.

Table 1: Pendulum: Actual Compuation Time (in second) Returned for specified different schemes.

simulation length period by

\begin{tabular}{|c|c|c|c|c|}
\hline \multirow{2}{*}{$\mathrm{S} / \mathrm{N}$} & \multirow{2}{*}{ Scheme } & \multicolumn{3}{|c|}{ Actual Computation Time for 3-simulation length periods } \\
\cline { 3 - 5 } & $\mathrm{R}=15000$ & $\mathrm{Tp}=25000$ & $\mathrm{Tp}=35000$ \\
\hline 1 & $\mathrm{RK} 2$ & 3 & 5 & 7 \\
\hline 2 & $\mathrm{RK}$ & 5 & 10 & 11 \\
\hline 3 & $\mathrm{RK} 4$ & 6 & 16 & 14 \\
\hline 4 & $\mathrm{RK5}$ & 9 & 17 & 23 \\
\hline 5 & $\mathrm{RK5M}$ & 10 & 12 & 17 \\
\hline 6 & RKB & 8 & & \\
\hline
\end{tabular}

Table 2: Duffing: Actual Compuation Time (in second) Returned for specified simulation length period by different schemes.

\begin{tabular}{|c|c|c|c|c|}
\hline \multirow[b]{2}{*}{$\mathrm{S} / \mathrm{N}$} & \multirow{2}{*}{ Scheme } & \multicolumn{3}{|c|}{ Actual Computation Time for 3-simulation length periods } \\
\hline & & $T p=15000$ & $\mathrm{Tp}=25000$ & $T p=35000$ \\
\hline 1 & RK2 & 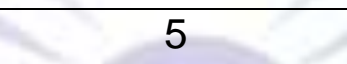 & 8 & 11 \\
\hline 2 & RK3 & +2 & 12 & 16 \\
\hline 3 & RK4 & 9 & 15 & 21 \\
\hline 4 & RK5 & 14 & 23 & 33 \\
\hline 5 & RK5M & 15 & 25 & 33 \\
\hline 6 & RKB & 10 & 18 & 25 \\
\hline
\end{tabular}

Tables 1 and 2 refers. The actual computation time increases non-uniformly from second order Runge-Kutta scheme to Butchers' (1964) modified fifth order schem and suffered dercrease on blended scheme for both Pendulum and Duffing oscillators. The actual computation time required for the simulation of the dynamics of pendulum is in the range (323seconds) while for the Duffing the range is (5-33seconds). The pendulum recorded higher actual computation time consistently compared with its Duffing counterpart and for corresponding cases. The range of the difference in actual computation time is (2-10seconds). 
Figures 3 to 5 below gives the variation of the normalised computation time (N-CT) with different schemes. The base value is the actual time reported for the second order scheme (RK2).

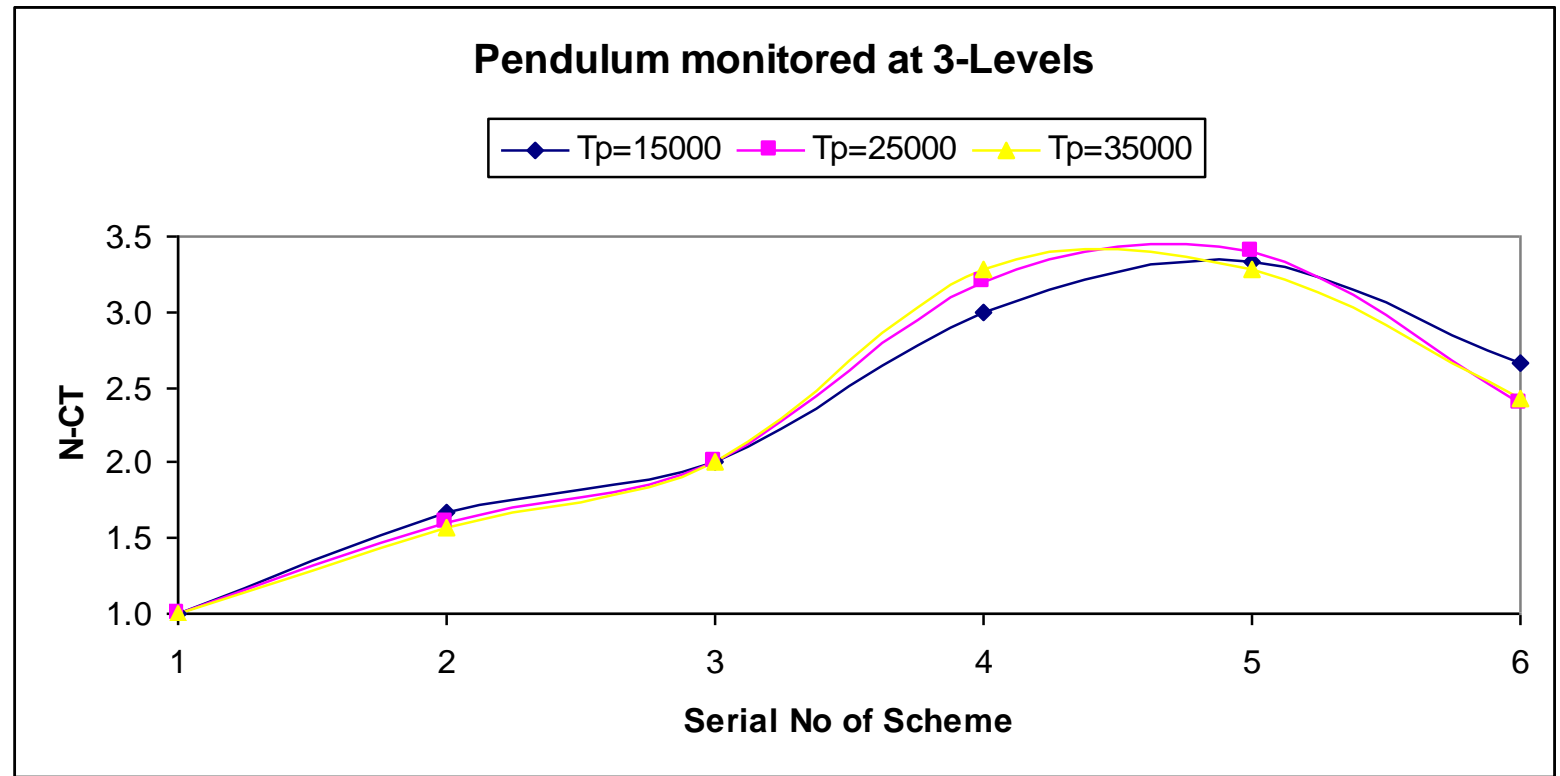

Figure 3: The variation of normalised computation time (N-CT) with the schemes for the case of pendulum.

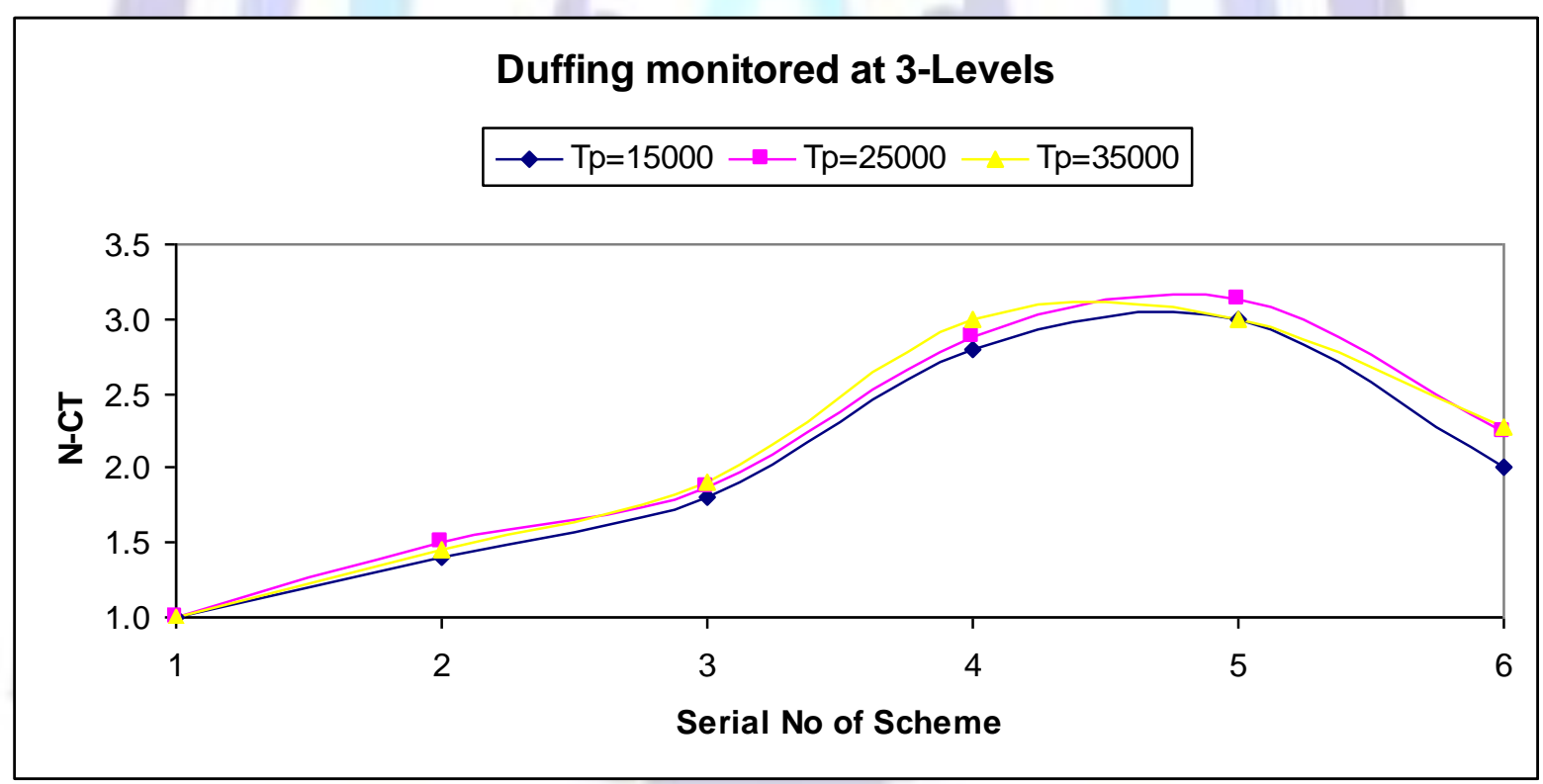

Figure 4: The variation of normalised computation time (N-CT) with the schemes for the case of Duffing. 


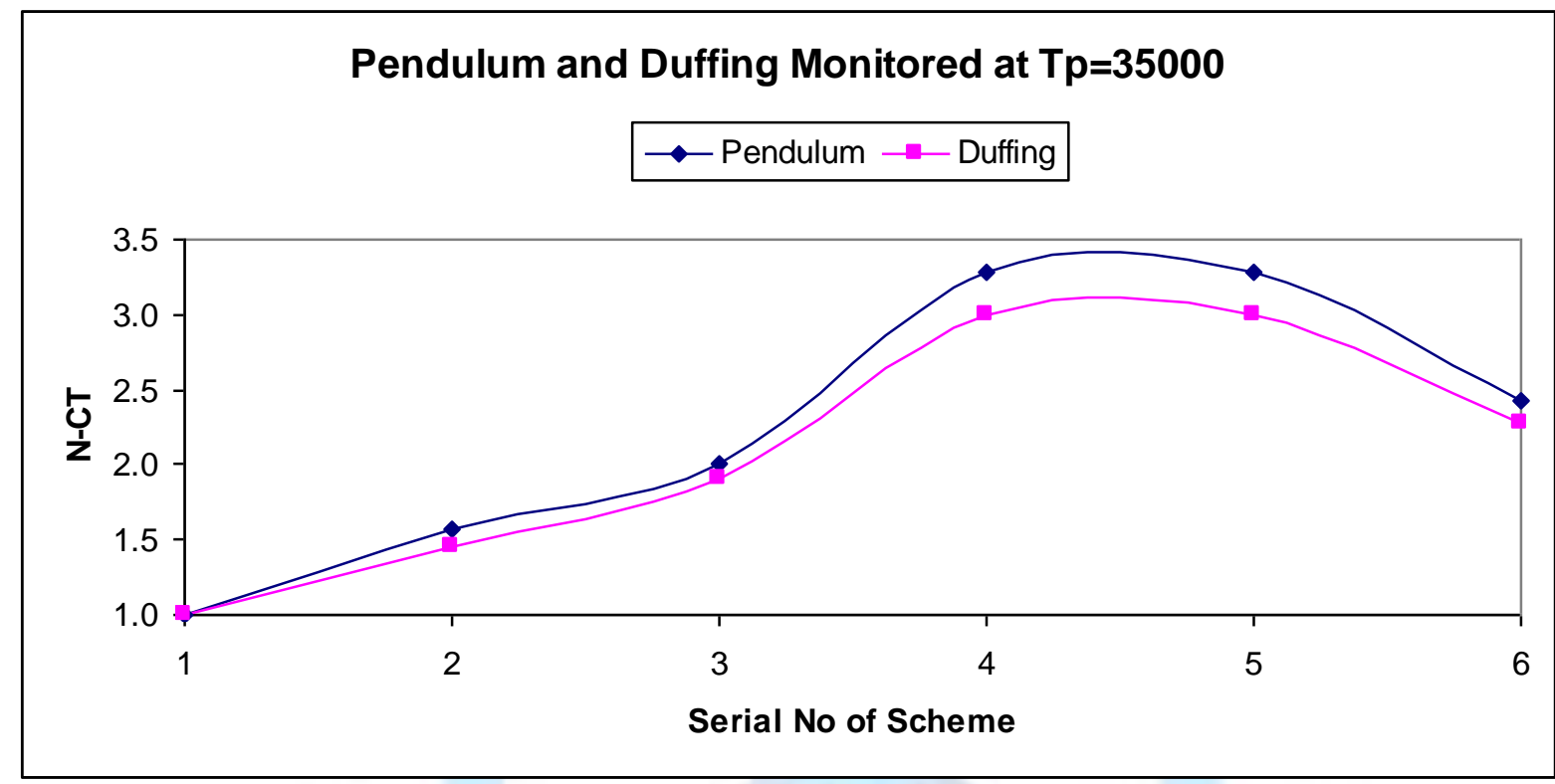

Figure 5: TComparison of the variation of normalised computation time $(\mathrm{N}-\mathrm{CT})$ with the schemes between Pendulum and Duffing.

Figures 3 to 5 refer. The trend of the normalised computation time in figure 3 suggest gravitation toward a well defined limit with incraesing simulation length period. Comparing figures 3 and 4 there is a repeat of observation made with the trend of the normalised computation time in figure 4. That is the variation trend indicate that a definite limit is attainable with increasing simulation length period. However figure 5 compare the variation of normalised computation time between two dynamics (Pendulum and Duffing) at large simulation length period of 35000 . It can be observed that insignificant difference exist between the corresponding normalised computation time as the scheme is varied. The average ratio of the normalised computation time for the schemes is $\{(1.0):(1.5):(2.0):(3.1):(3.1):(2.4)$ at $\mathrm{Tp}=3500$ for Rk2, RK3, RK4,RK5, RK5M and RKB respectively. Thus the ratio can be said to be insentive or do not have regard for the dynamics system being investigated.

\section{CONCLUSIONS}

This study has developed a simulation platform involving multiple Runge-Kutta schemes for the investigation of the required computational time for the cases of nonlinear and harmonically excited oscillators (pendulum and Duffing). The actual computation time required for the simulation of the dynamics of pendulum is in the range (3-23seconds) while for the Duffing the range is (5-33seconds). The pendulum recorded higher actual computation time consistently compared with its Duffing counterpart and for corresponding cases. The range of the difference in actual computation time is (210 seconds). Interestingly the normalised computation time required for corresponding cases are insensitive to system (pendulum or Duffing). Its average for the systems produced simplest ratio $\{(1.0):(1.5):(2.0):(3.1):(3.1):(2.4)\}$ at $\mathrm{Tp}=3500$ for the second, third, fourth, fifth, Butchers'(1964) modified fifth order schemes and their unfiform but randombased blend respectively. It is concluded that higher computation time factor associated with the hiher order Runge-Kutta schemes can be accomodated on the assurance of provison of accurate simulation results.

\section{REFERENCES}

(1) Chen L., Lou Q., Li Z. and Zhu W. (2012), Stochastic stability of the harmonically and randomly excited Duffing oscillator with damping modelled by a fractional derivative. Science China Physics Mechanics and Astronomy, Vol. 55, Issue 12, pp. 2284-2289. (C) Science China Press.

(2) Classweb (2013), Introduction to computer simulations. An article written by management science classweb downloaded from classweb.gmu.edu/aloerch/ simulations540.pdf on 20th July, 2013 at 3.13 PM.

(3) Diks C., Hommes C., Panchenko V. and Weide K.V. (2008), Economics and Finance: A user friendly software package for nonlinear economics dynamics. Computer Economics, Vol. 32, pp. 221-244, Springer.

(4) Dowell E.H. (1988), Chaotic oscillations in mechanical systems, Computational Mechanics, 3, pp.199-216.

(5) Finger L. and Uhlmann H. (1994), Effective computation of the Poincaré map for the analysis of nonlinear dynamic circuits/systems using Runge-Kutta triples. Journal of circuits, systems and computers, Vol.4, Issue 1. (c) 2013 World Scientific Publishing.

(6) Gregory L. B. and Jerry P. G. (1990), Chaotic dynamics: An Introduction, Cambridge University Press, USA, pp.3-5 \& 40-75.

(7) Haecheon C. and Parviz M. (1994), Effects of the computational time step on numerical solutions of turbulent flow. Elsevier Journal of Computational Physics.Vol.113, Issue 1, pp.1-4. 
(8) Ivana K., Michael J.B. and Asok K.M. (2011), Forced vibration of a Duffing oscillator with different damping mechanisms. Wiley online library. DOI: 10.1002/9780470977859.Ch6. (c) 2011 John Wiley and Sons Ltd.

(9) Jian Z. and Huazhong T. (2013), Runge-Kutta discontinuous Galerkin methods with WENO limiter for the special relativistic hydrodynamics. Journal of computational physics, Vol.242, pp. 138-168. Academic Press Professional, Inc., San Diego, CA ,USA.

(10) Liang Y. and Feeny B.F. (2008), Parametric identification of a chaotic based excited double pendulum experiment. Springer Journal of Nonlinear Dynamics, Vol. 52, pp. 181-197. @ Springer Science + Business Media, Inc.

(11) Ludovic N., Laurent S., Jean P.P. and Jérome B. (2002), Automatic time algorithms for implicit numerical simulations of nonlinear dynamics. Advances in Engineering Software: Engineering computational Technology, Vol. 33, Issue 7-10, pp. 589-603, Elsevier Science Ltd., UK.

(12) Murugesh V., Murugesan K. and Kim K. (2011), Simulation of nonlinear singular system using RK-Bucther Algorithm. ICHIT 2011, CCIS 206, pp.625-632.@ Springer-Verlag Berlin Heidelberg 2011

(13) Mustafa M., Mada S.W.S and Dian S.M. (2013), Numerical simulation of chaotic synchronization of Chua circuit and its applications for secure communications. Applied Mathematical Sciences, Vol. 7, No. 1, pp.1-10.

(14) Nikolaos S. (2009), An algorithm using Runge-Kutta methods of orders 4 and 5 for systems of ODEs. International Journal of Numerical Methods and Applications, Vol.2, No.1,pp. 47-57. (c) 2009 Pushpa Publishing House.

(15) Salau T.A.O. and Ajide O.O. (2012), Comparative analysis of time steps distribution in Runge-Kutta algorithms. International Journal of Scientific and Engineering Research (IJSER). Vol. 3, Issue1, pp.1-5. ISSN 2229-5518.

(16) Salau T.A.O. and Ajide O.O. (2013), A novel graphic presentation and fractal characterization of Poincaré solutions of harmonically excited pendulum. International Journal of Advances in Engineering and Technology (IJAET), Vol.6, Issue 3, pp. 1299-1312.

(17) Salau T.A.O. and Ajide O.O.(2013), Runge-Kutta schemes coefficient simulation for comparison and visual effects. SCIRP Engineering Journal, Vol.5, No.5, pp. 530-536.Scintific Research, USA

(18) Seth P. (2013), Improving simulation performance in simulink. (C) 1994-2013 The Mathworks Inc.

(19) Siddhartha K.K. and James D.M. (2008), Multifractal solver for online power system time-domain simulation. IEEE Transactions on power systems, Vol. 23, No. 4, pp. 1727-1737.

(20) Steven C. C. and Raymond P. C. (2006), Numerical methods for engineers, Fifth edition, McGraw-Hill (International edition), New York, ISBN 007-124429-8.

(21) Wang J. (2013), He's Max-Min approach for coupled cubic nonlinear equations arising in packaging system Hindawi Journal: Mathematical problems in Engineering, Vol. 2013, 4 pages. Article ID: 382509

Appendix: Structural Outline of Simulation Procedures

(1) Start

(2) Read input data including the prescribed system (pendulum or Duffing) parameters, initial condition, simulation period lengths (unsteady and steady), number of simulation time step per period and random numer generating seed value.

(3) Perform the preliminary calculations that is common to all Runge-Kutta schemes to be investigated.

(4) Utilised second order Runge-Kutta scheme to simulate the dynamic system (Pendulum or Duffing) from the precribed initial conditions to the end of specified simulation length period. As the simulation progress monitor the actual computaation time at three simulation periods specified using current time call subroutine command and track results for further processing.

(5) Repeat (4) for the third order scheme.

(6) Repeat (4) for the fourth order scheme

(7) Repeat (4) for the fifth order scheme

(8) Repeat (4) for the Butchers' (1964) modified fifth order scheme.

(9) Repeat (4), but make selection of scheme at random from the lists of five: second, third, fourth, fifth and Butchers' (1964) modified fifth order schemes.

(10) Stop and End.

(11) Perform relevants post simulation processing of the output results using Microsoft Excel-2003. 